\title{
OPEN ACTIVE CONTOUR MODEL FOR FRONT TRACKING OF DETONATION WAVES
}

\author{
Samee Maharjan $^{1} \quad$ Andre Vagner Gaathaug $^{1} \quad$ Ola Marius Lysaker $^{1}$ \\ ${ }^{1}$ Department of process, energy and environmental technology, University College of Southeast Norway, Norway. \\ samee. maharjan@usn. no
}

\begin{abstract}
This paper presents an image processing framework for tracking the front of the detonation wave from a sequence of images. The images are captured by high speed camera during a laboratory gas explosion experiment. By tracking the fronts in two or three consecutive frames, it is possible to calculate the thermodynamic properties like velocity and pressure along the entire wave front. Alternatively, these calculations are limited to measurements recorded by sensors at some fixed, locations. An active contour model having Gradient Vector Flow (GVF) as an external force field is used to track the wave front in each image. The structure and the properties of detonations in combustion physics has been the point of interest since early 80's. In the present paper, detonation is studied in the stratified layer of combustible gas above a non-reacting layer of air. The recorded images are digitally processed, and the local velocities are calculated based on the tracked fronts. The calculated velocities are then used to estimate the pressure ahead of the wave front with the help of the normal shock relations. The estimated pressure is compared with the measured values from pressure transducers mounted on the top and bottom of the experiment tube.
\end{abstract}

\section{Keywords: Open contour, Front Tracking, Detonation}

\section{Introduction}

Active contours, popularly known as snakes, have been an essential part in image processing and computer vision applications. Snakes are mainly used for edge detection and boundary contouring in the field of image segmentation. A snake is a moving curve within an image, which eventually lie itself around the surface/edge of the desired object. The snake moves under the influence of internal forces within the curve itself and the external force calculated from the image data. It was first developed by Kass et al.in 1988 (Kass et al., 1988). Many developments and improvements have been purposed thereafter, for example the Chan-Vese (CV) model (Chan and L.Vese, 2001) that detects the boundary of an object using the MumfordShah functional (Mumford and Shah, 1989), the level set method (Osher and Sethian, 1988) to name a few. The application of active contours are seen mainly in the field of medical research see, (Phama and Tranc., 2015; Yan et al., 2014) and the references therein. For the last decade, active contour models have been an active research field. However most applications are based on closed contours. But for some application like front tracking, where the edge/boundary expands from top to bottom of the image an open snake is more efficient. This study will show the development of an open contour model and its application in the field of physical science of detonations.

Detonations are among the worst consequences of accidents related to gas handling and explosion in which a generated wave can exert pressure around 40 bar and velocity of more than $2000 \mathrm{~m} / \mathrm{s}$. It is relevant for many combustible gases, while mostly considered for the more reactive gases such as propane, ethylene, hydrogen and acetylene (Law, 2010). The detonation study of this work regards the detonations in a stratified layer of hydrogen gas above a non-reacting layer of air. The formation of a high-pressure Mach stem at the lower wall (see Figure 4) is of particular interest in the field of Combustion.

The paper is organized as follows: In Section 2, there is a short description of the experimental setup that was used for the experiment and image recording. In Section 3 , a general snake model is described which is followed by Section 4, where the numerical implementation of an open snake is shown. The front tracking and velocity calculation is put together in Section 5. Finally, the results and the conclusions of the study is presented in Section 6.

\section{Experimental Setup}

Figure 1 illustrates the experimental setup for conduting the gas explosion experiments. The setup is made of a $3 \mathrm{~m}$ long channel with transparent polycarbonate walls. One end is closed and the other is open to the atmosphere, and an adjustable baffle type obstacle is located at $1 \mathrm{~m}$ from the closed end. The channel was filled with hydrogen and air and then ignited from the closed end such that the wave propagated towards the open end. Kistler 603b type pressure transducers were used to record the pressure at various locations along the top and the bottom wall of the tube. A high speed camera recorded the wave propagation behind the obstacle at $500 \mathrm{kHz}$. A schlieren imaging method was used to record the propagation at $23 \mathrm{~cm}$ diameter window expanding from a $1.2 \mathrm{~m}$ to 1.4 $m$ behind the obstacle. The schlieren system is a double mirror system which is based on the fact that light rays 
bends whenever there is a change in the refractive index of the meduim in which the light passed (Settles, 2001). All experiments are done with premixed hydrogen and air at ambient temperature and pressure. Detail description of the setup can be found in (Gaathaug et al., 2010).

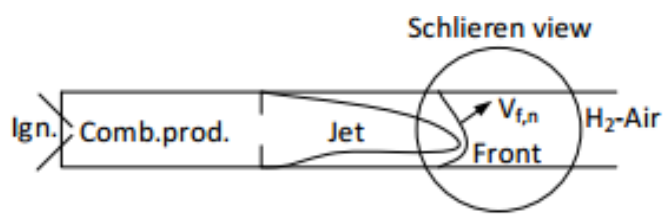

Figure 1. Experimental setup for premixed hydrogen and air combustion in a long tube.

Figure 2(a) shows the portion of captured high speed frame sequence during an experiment and Figure 2(b) shows the schlieren images captured at the highlighted rectangle portion in Figure 2(a). An overview of the over-
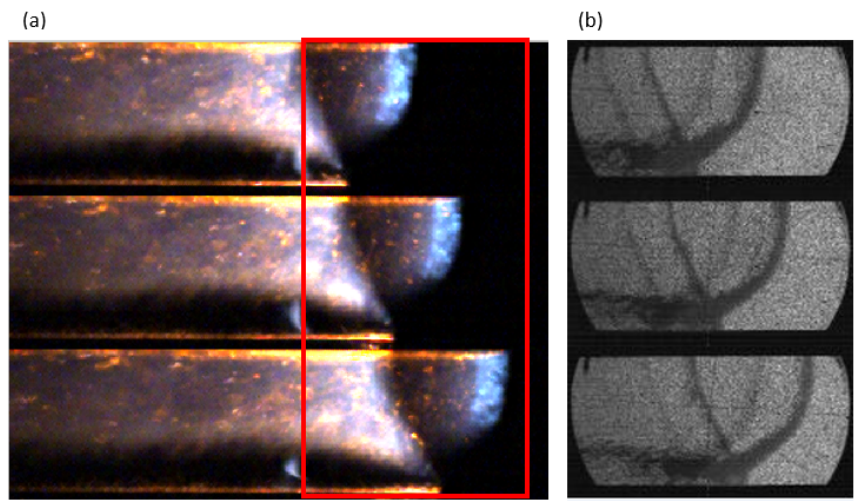

Figure 2. (a) High speed images showing a detonation wave in an experiment tube.(b) The schlieren images captured within the red rectangle in (a).

all designed framework for tracking the fronts from the image is depicted in the flowchart shown in Figure 3.

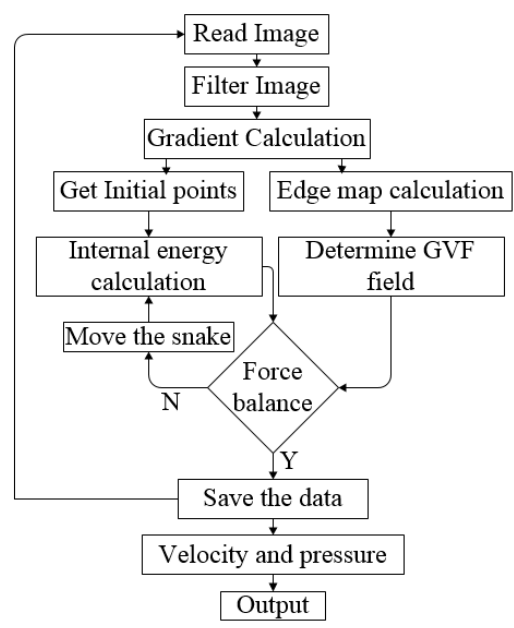

Figure 3. Flow chart showing the designed framework.

\section{Active Contour Model}

A basic snake defined in (Kass et al., 1988) is a parametric curve formed within an image. The snake is initialized manually by a set of $x-y$ points around the object of interest and simultaneous interpolates between the points. By representing the position $s$ of the snake parametrically as $V(s)=(x(s), y(s))$ in $\mathrm{x}$-y coordinate system , its energy function is defined as in (1). The energy is calculated for each point along the snake and the snake will move in the direction where there is minimum energy compare to the previous position. The snake will be stationary once the energy is balanced in all directions.

$$
E_{\text {snake }}=\int_{s} E_{\text {int }}(V(s))+E_{\text {ext }}(V(s)) d s .
$$

The first term in (1), $E_{\text {int }}$ is an internal energy which can be defined as

$$
E_{\text {int }}=1 / 2\left[\alpha(s)\left|V^{\prime}(s)\right|^{2}+\beta(s)\left|V^{\prime \prime}(s)\right|^{2}\right] .
$$

Here $\alpha(s)$ and $\beta(s)$ are positive weighting parameters for controlling the snake's tension and rigidity respectively. $V^{\prime}(s)$ and $V^{\prime \prime}(s)$ are the first and the second derivative of $V(s)$ with respect to $s$. The second term in (1), $E_{\text {ext }}$ is the external energy function which is calculated from the image such that it takes minimum values at the point of interest like edges and boundaries. For example, for a gray scale image $\operatorname{Im}(x, y)$, the external energy can be calculated as

$$
E_{\text {ext }}=-|\nabla \operatorname{Im}(x, y)|^{2},
$$

where $\nabla$ is the gradient operator. A snake that minimizes $E_{\text {snake }}$ must also satisfy the Euler equation

$$
\alpha V^{\prime \prime}(s)-\beta V^{\prime \prime \prime \prime}(s)-\nabla E_{e x t}=0,
$$

which can be viewed as force balance between the internal and the external forces. Both $\alpha(s)$ and $\beta(s)$ are taken as constant for the entire framework. Mathematically,

$$
F_{\text {int }}+F_{\text {ext }}=0 .
$$

The internal force term $F_{\text {int }}=\alpha V^{\prime \prime}(s)-\beta V^{\prime \prime \prime \prime}(s)$ prevents the snake from stretching and bending whereas the external force term $F_{e x t}=-\nabla E_{e x t}$ attracts the snake towards the desired location (Xu and Prince, 1997).

To solve (4), $V(s)$ is taken also as a function of time $t$ such that $V(s)$ moves with time step $t$ i.e. $V(s, t)$. The external energy $E_{e x t}$ for the image does not change with time.

$$
\frac{\partial V(s, t)}{\partial t}=\alpha V^{\prime \prime}(s, t)-\beta V^{\prime \prime \prime \prime}(s, t)-\nabla E_{e x t} .
$$




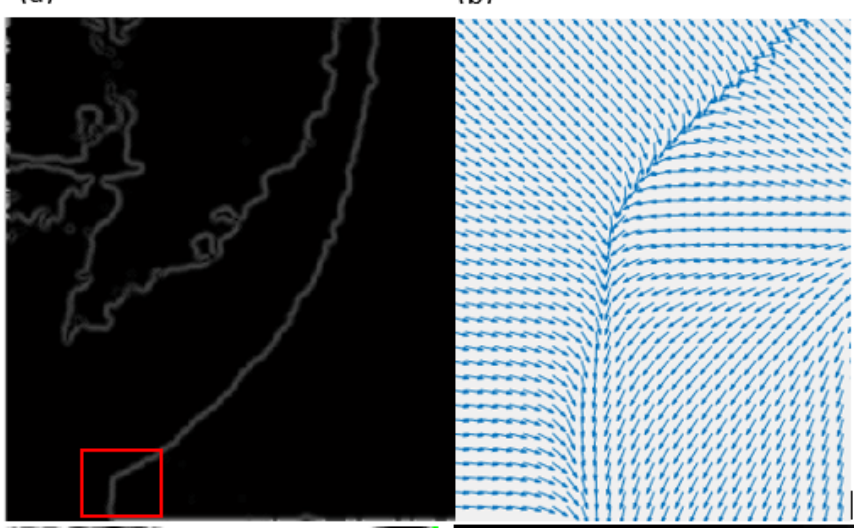

Figure 4. (a) Edge map of the image, Mach stem area is highlighted with red square. (b) External force field around the Mach stem.

\section{Numerical Implementation}

The numerical solution of (6) can be found when $\frac{\partial V(s, t)}{\partial t}$ tends to zero and (6) becomes,

$$
\alpha V^{\prime \prime}(s, t)-\beta V^{\prime \prime \prime \prime}(s, t)-\nabla E_{e x t}=0 .
$$

The solution for (7) can be obtained by decritizing the continous snake $V(s, t)$ as in (8). The details can be found in (Kass et al., 1988).

$$
\begin{array}{r}
\alpha\left[V_{i}-V_{(i-1)}\right]-\alpha\left[V_{(i+1)}-V_{i}\right]+\beta\left[V_{(i-2)}-2 V_{(i-1)}+V_{i}\right] \\
-2 \beta\left[V_{(i-1)}-2 V_{i}+V_{(i+1)}\right]+\beta\left[V_{i}-2 V_{(i+1)}+V_{(i+2)}\right] \\
+\left(f_{x}(i), f_{y}(i)\right)=0 .
\end{array}
$$

Here $V_{i}=\left(x_{i}, y_{i}\right)$ is the $i^{t h}$ point of the contour/snake $s$ and $\left(f_{x}(i), f_{y}(i)\right)$ which are equal to $\left(-\partial E_{\text {ext }} / \partial x_{i}\right.$, $\left.-\partial E_{e x t} / \partial y_{i}\right)$ are the external force at point $i$.

\subsection{Internal energy}

The overall internal energy at each point can now be described in a matrix form as

$$
A V+\left(f_{x}(i), f_{y}(i)\right)=0 .
$$

Here $A$ is pentadiagonal matrix ,

$$
A=\left[\begin{array}{ccccc}
\beta & 0 & 0 & 0 & 0 \\
0 & \alpha-3 \beta & 0 & 0 & 0 \\
0 & 0 & 2 \alpha+6 \beta & 0 & 0 \\
0 & 0 & 0 & -\alpha-4 \beta & 0 \\
0 & 0 & 0 & 0 & \beta
\end{array}\right]
$$

and $V$ is a vector of the 5 consecutive points, $V=\left[\begin{array}{lllll}V_{i-2} & V_{i-1} & V_{i} & V_{i+1} & V_{i+2}\end{array}\right]^{T}$.
If $n$ is the total number of points in each contour, then for closed contour $V_{1}=V_{n}$, the internal energy can be estimated using (8) for all points along the contour. However, for open contours the end points should remain at the top and bottom boundary, hence $V_{(i-1)}$ and $V_{(i-2)}$ will not exists for the first two points whereas $V_{(i+1)}$ and $V_{(i+2)}$ for the last two points. The matlab program is designed in such a way that these points stays at the boundary and therefore only move in $x$-direction with respect to its own external energy and its own previous position. Using Euler method with time step $t$ for (8), the contour point $V_{i}^{t}$ at time $t$ can be related to its previous point $V_{i}^{t-1}$ as,

$$
A V_{i}^{t}+\left(f_{x}(i), f_{y}(i)\right) V_{i}^{t-1}=-\gamma\left(V_{i}^{t}-V_{i}^{t-1}\right)
$$

where $\gamma$ is a step size. Solving (10) by matrix inversion and separating $V_{i}$ into $x_{i}$ and $y_{i}$,

$$
\begin{aligned}
& x_{i}^{t}=(A+\gamma I)^{-1}\left[\gamma x_{i}^{(t-1)}-f_{x}\left(x_{i}^{(t-1)}, y_{i}^{(t-1)}\right]\right. \\
& y_{i}^{t}=(A+\gamma I)^{-1}\left[\gamma y_{i}^{(t-1)}-f_{y}\left(x_{i}^{(t-1)}, y_{i}^{(t-1)}\right],\right.
\end{aligned}
$$

where $I$ is an identity matrix.

\subsection{External energy}

One of the main drawback of the snake models is that the calculated external energy were not sufficient for attracting the snake from long distance. The simple external energy calculated by using (3) works nicely when the initial snake is near to the object but fails to attract the snake when it is placed far away. To overcome this drawback, the gradient vector flow (GVF) proposed in (Xu and Prince, 1997) is used for the external force field. For estimating GVF, first the edge map $E_{\text {edge }}(x, y)$ is formed in such a way that it has a larger value at the edges and at the boundaries, compared to homogeneous region. Later, the estimated edge map is used to estimate the over all external force field. For this study, the edge map is taken as the gradient magnitude of the image,

$$
E_{\text {edge }}(x, y)=\sqrt{\left(g_{x}^{2}+g_{y}^{2}\right)}, \quad\left[g_{x}, g_{y}\right]=\nabla \operatorname{Im}(x, y) .
$$

and the result in shown in Figure 4(a). The Mach stem is marked by a red rectangle in this figure. Following the steps described (Xu and Prince, 1997), the final external external force $\left(f_{x}(i), f_{y}(i)\right)$ for an image is calculated by iterating the equations (13) and (14) until it converges.

$$
f_{x}(i)=\mu \nabla^{2} f_{x}(i)-\left|\nabla E_{\text {edge }}(x)\right|^{2}\left(f_{x}(i)-\nabla E_{\text {edge }}(x)\right)
$$

$$
f_{y}(i)=\mu \nabla^{2} f_{y}(i)-\left|\nabla E_{\text {edge }}(y)\right|^{2}\left(f_{y}(i)-\nabla E_{\text {edge }}(y)\right)
$$


(a)

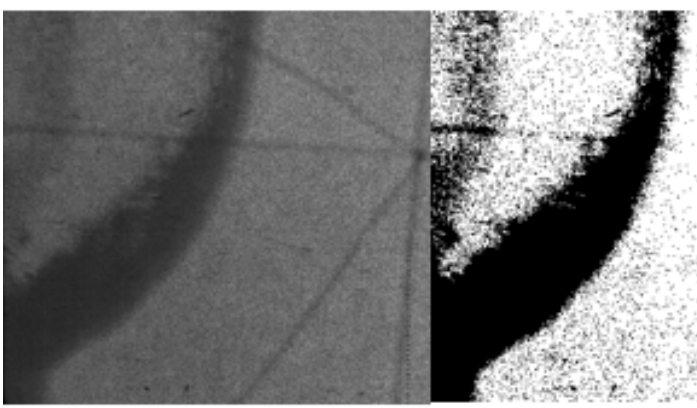

(c)

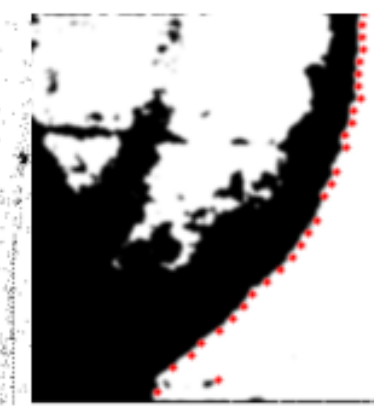

(d)

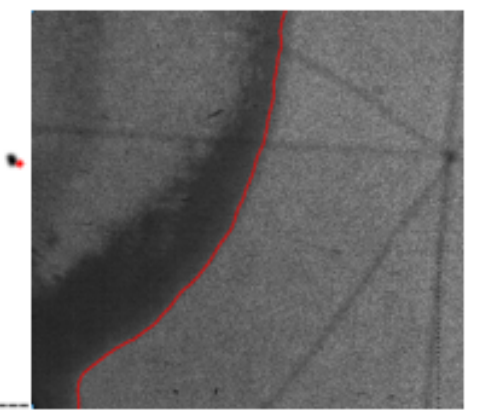

Figure 5. (a) Original raw image (b) Binary image (c) Filtered binary image with initial points for the snake. (d) Final contour along the wave front.

Here $\nabla^{2}$ is the lapalcian operator and $\mu$ is a regularization parameter governing the trade off between the first term and second term. Generally, $\mu$ is taken as a constant which is set according to the noise present in the image. For a sequence of images where the noise varies with time, $\mu$ along with $\left|\nabla E_{\text {edge }}(x, y)\right|^{2}$ can be taken as spatially varying weighting factor as presented in $(\mathrm{Xu}$ and Prince, 1998). The initial value for $\left(f_{x}(i), f_{y}(i)\right)$ is taken as $\nabla E_{\text {edge }}(x, y)$. The computed GVF field points towards the edges and varies smoothly over homogeneous regions, see Figure 4(b).

\section{Front tracking and velocity calcula- tion}

It is necessary to reduce the background noise presents in the images before actual tracking of the front. For this purpose, the pre-processing of the image is done by first changing each image into binary form using Otsu method (Otsu, 1976), follwed by filtering using median filter. Figure 5(a) shows the raw image from the experiment and in Figure $5(\mathrm{~b})$ the binary version of the same image is visualized. Though the GVF force field has advantages compared to the external forces used in (Kass et al., 1988), the formation of the initial points for the snake highly influence the overall performance of the framework. One possible way to initialize the snake automatically to a location close to the object, is to use the information of the gradient values in the image. For this task, a priori information of the direction of the wave propagation is used. By starting a search from the opposite direction of the wave propagation for predefined set of rows (every $20^{t h}$ ), an initial point is chosen to be the first point where the gradient value exceeds a given threshold value, see Figure 5(c). The snake is then initialized by interpolating these initial points such that there exist the snake point $V(s)$ for each unique row value of the image. For example, if size of an image $I m$ is $[300 \times 800]$ then the size of the snake is $[300 \times 1]$. By implementating, the method defined in Section 4, the final contour is obtained as shown in Figure 5(d).

Suppose $V^{K}(s)$ is the final snake (tracked front) in $K^{t h}$ image of the sequence. For calculation of a normal ve- locity at point $V_{i}=\left(x_{i}, y_{i}\right)$ of the front $V^{K}(s)$, a local normal vector $\vec{n}$ is calculated by forming a local polynomial function around the point $V_{i}$ (5 points above and 5 points below are used). The normal vector $\vec{n}$ will forms a triangle between the fronts with an angle $\theta$ at point $V_{i}$. The normal displacement is now estimated by using the final snake of the $V^{K+1}(s)$ of $(K+1)^{t h}$ image of the sequence as shown in Figure 6. By using the value of $V^{K+1}(s)$ for the same row $y_{i}$ the displacement $d_{m}$ can be calculated and an angle $\theta$ can be estimated by calculating the slope of the normal vector $\vec{n}$.

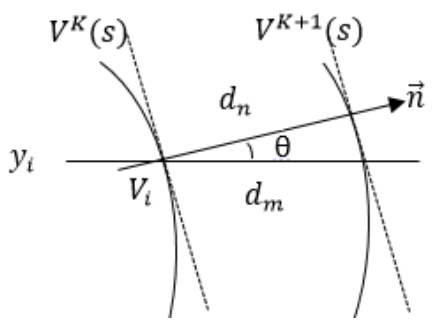

Figure 6. Method for estimating normal front velocity.

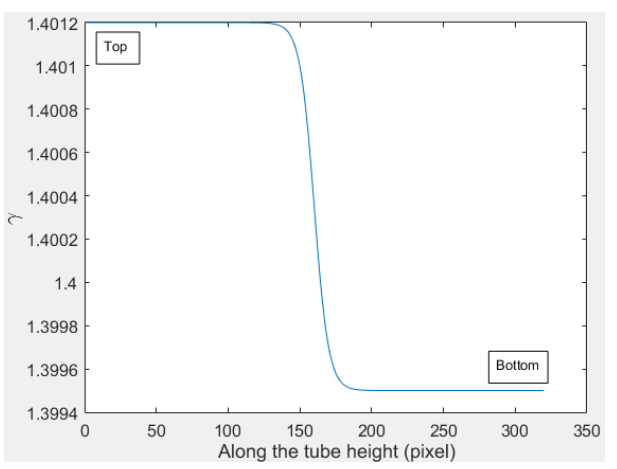

Figure 7. Spatially varying $\gamma$ for top and bottom of the tube.

By assuming the normal vector $\vec{n}$ is locally normal to both fronts at $K$ and $K+1$, a normal displacement $d_{n}$ is estimated as $d_{n}=d_{m} * \cos \theta$.

The normal displacement $d_{n}$ is then changed to standard unit of meter with system configuration of $(1 p x=$ $0.0002778 \mathrm{~m}$ ) and the normal velocity $V_{f}$ is calculated at 
(a)

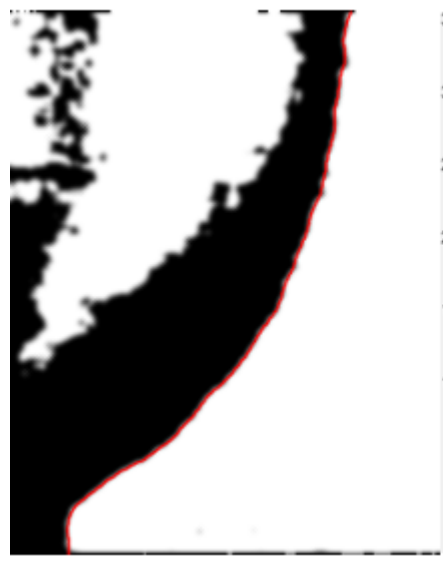

(b)

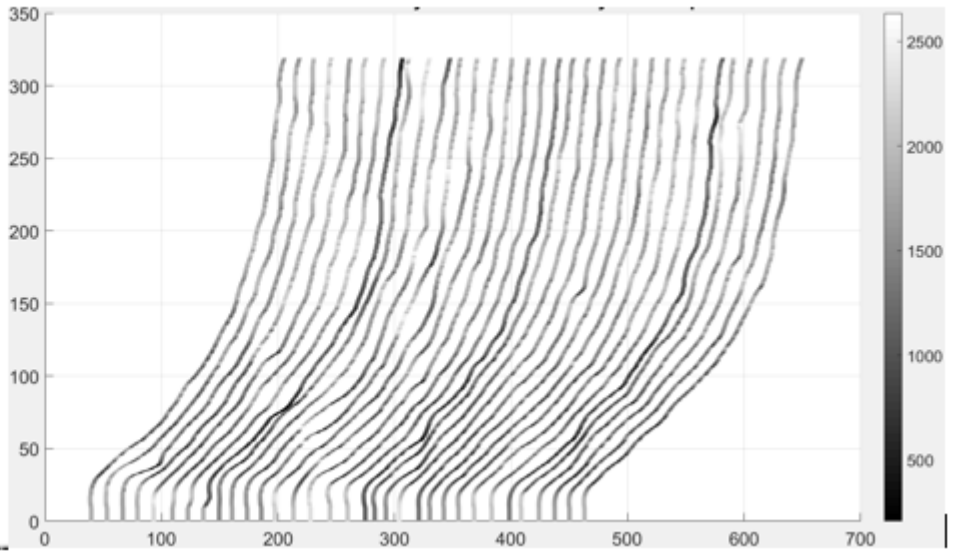

Figure 8. (a)A final contour in filtered binary image.(b) The front contours with local velocity (m/s) given by the color according to the colormap.

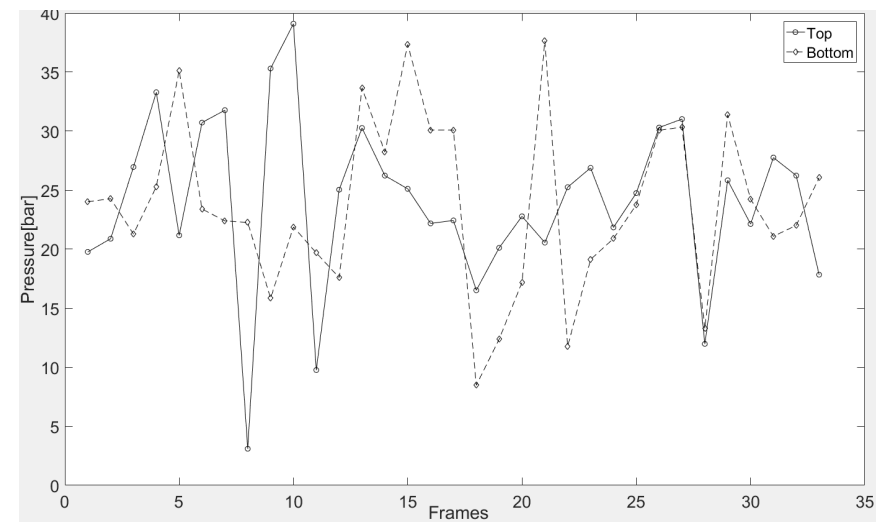

Figure 9. Pressure estimated for top and bottom of each front.

frequency of $500 \mathrm{kHz}$. By using the calculated velocity to estimate the Mach number $M$, the pressure behind the front is estimated by normal shock relation shown in (15) (Law, 2010).

$$
\frac{P_{1}}{P_{0}}=\frac{2 \gamma M^{2}-(\gamma-1)}{\gamma+1} .
$$

Here, $P_{1}$ is the pressure behind of the wave front and $P_{0}$ is the pressure ahead the wave front. The pressure ahead of the wave front is assumed to be 1 bar in this case. The Mach number $M$ is defined as,

$$
M=\frac{V_{f}}{c},
$$

$c=\sqrt{\gamma R T / M}$ is the speed of sound in medium $\left(\mathrm{H}_{2}\right)$ at temperature $T . R, M$ and $\gamma$ are respectively gas constant, Molecular mass and specific heat ratio of $\mathrm{H}_{2}$. As the gas is different in top and bottom of the tube, $\gamma$ is taken as spatially varying along the tube from 1.3995 to 1.4012 as shown in Figure 7 ( $\gamma$ for $H_{2}$ is 1.4). The speed of sound for hydrogen is calculated and considering temperature $T$ ahead the wave front is uniform at $25^{\circ} \mathrm{C}$. The estimated $M$ is then used for finding pressure $P_{1}$ from (15).

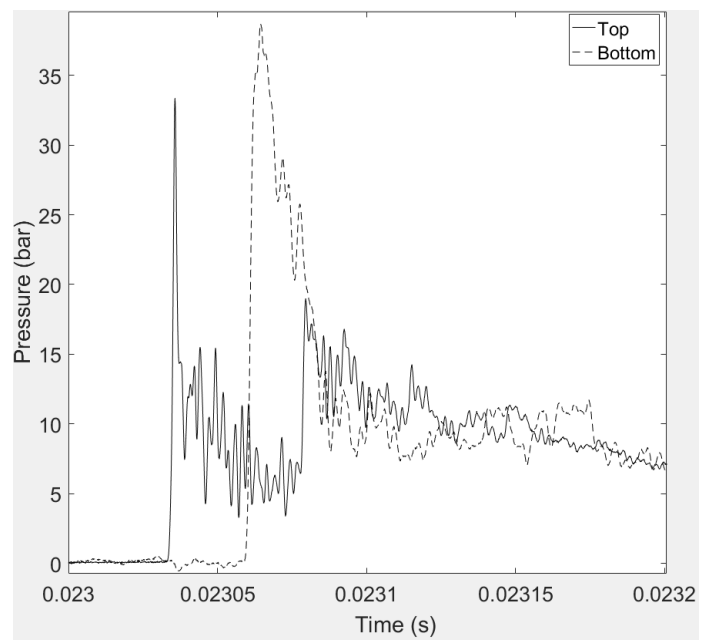

Figure 10. Pressure records from the experiment by the pressure transducers.

\section{Results and Conclusion}

For clarity, the final contour along the detonation wave front is plotted in filtered image shown in Figure 8(a). The calculated velocities along all the tracked wave fronts from a sequence is plotted in Figure 8(b). As expected the velocity in the upper layer that is filled with combustible gas is relatively higher than the velocity in the bottom layer. Due to the generation of Mach stem along the lower boundary of the tube, velocity of the front around the lower boundary is noticeably high. The average pressure estimated at top and bottom of the each front is plotted in Figure 9 ( top and bottom 10 points are used). The reading from pressure transducers mounted on the top and the bottom of the tube at fixed location is shown in Figure 10. Due to the difference between the location of pressure transducers and the moving wave front, direct comparision cannot be justified. Nevertheless, it can be seen that maximum and minimum pressure on both the figure are almost in same range. 
The designed image processing framework was also used to calculate the velocity for a benchmark experiment. The results of the experiment shows an average detonation velocity of $1967 \mathrm{~m} / \mathrm{s}$ which is $9 \mathrm{~m} / \mathrm{s}$ lower than the theoretical value (Gaathaug et al., 2016). Thus, it can be assumed that the framework gained detailed information of the detonation front within expected precision. Further work need to be done considering assumptions made during pressure estimation using a normal shock relation.

\section{References}

Tony F. Chan and L.Vese. Active contours without edges. Image Processing, IEEE Transactions., 10:266-277, 2001.

Andre Vagner Gaathaug, Dag Bjerketvedt, and Knut Vaagsaether. Experiments with flame propagation in a channel with a single obstacle and premixed stoichiometric h2-air. Combustion Science and Technology., 182: 1693-1706, 2010.

Andre Vagner Gaathaug, Samee Maharjan, Ola Marius Lysaker, Knut Vaagsaether, and Dag Bjerketvedt. Velocity and pressure along detonation fronts - image processing of experimental results. Proc. of the Eighth International Seminar on Fire and Explosion Hazards (ISFEH8), 2016.

Michael Kass, Andrew Witkin, and Demetri Terzopoulos. Snakes: Active contour models. International Journal of Computer Vision, 1:321-331, 1988.

Chung K. Law. Combustion Physics. Cambridge University Press, New York, USA, 2010.

David Mumford and Jayant Shah. Optimal approximations by piecewise smooth functions and associated variational problems. Communications on Pure and Applied Mathematics., 42:577-685, 1989.

Stanley Osher and James A. Sethian. Fronts propagating with curvature dependent speed: Algorithms based on hamiltonjacobi formulations. Journal of Computational Physics., 79: 12-49, 1988.

Nobuyuki Otsu. A threshold selection method from gray-level histograms. IEEE Transactions on Systems, Man, and Cybernetics., 9:62-66, 1976.

Van-Truong Phama and Thi-Thao Tranc. Active contour model and nonlinear shape priors with application to left ventricle segmentation in cardiac mr images. Optik - International Journal for Light and Electron Optics, 127:991-1002, 2015.

Gary S. Settles. Schlieren and Shadowgraph Techniques: Visualizing Phenomena in Transparent Media. Springer-Verlag Berlin Heidelberg New York, 2001. ISBN 0.

Chenyang Xu and Jerry L. Prince. Gradient vector flow: A new external force for snakes. IEEE Proc. Conf. on Computer vision and Pattern Recognigition, pages 66-71, 1997.

Chenyang $\mathrm{Xu}$ and Jerry L. Prince. Generalized gradient vector flow external forces for active contours. Signal Processing., 71:131-139, 1998.
Zhoua Yan, Ren Shia Wei, Wei Chenc, Yong lin Chenc, Ying Lid, Li-Wen Tand, and Dai-Qiang Chenb. Active contours driven by localizing region and edge-based intensity fitting energy with application to segmentation of the left ventricle in cardiac ct images. Neurocomputing, 156:199-210, 2014. 